BMJ Open

Sport \&

Exercise

Medicine

\title{
Self-reported physical activity before a COVID-19 'lockdown': is it just a matter of opinion?
}

\author{
Troy J Cross (10 , ${ }^{1,2}$ Jennifer M J Isautier, ${ }^{3}$ Emmanuel Stamatakis, ${ }^{1}$ \\ Sarah Jane Morris, ${ }^{1}$ Bruce D Johnson, ${ }^{2}$ Courtney Wheatley-Guy, ${ }^{4}$ Bryan J Taylor ${ }^{5}$
}

To cite: Cross TJ, Isautier JMJ, Stamatakis E, et al. Selfreported physical activity before a COVID-19 'lockdown': is it just a matter of opinion? BMJ Open Sport \& Exercise Medicine 2021;7:e01088. doi:10.1136/ bmjsem-2021-001088
Check for updates

\section{(c) Author(s) (or their} employer(s)) 2021. Re-use permitted under CC BY-NC. No commercial re-use. See rights and permissions. Published by BMJ

${ }^{1}$ Faculty of Medicine and Health, The University of Sydney, Sydney, New South Wales, Australia

${ }^{2}$ Cardiovascular Medicine, Mayo Clinic, Rochester, Minnesota, USA

${ }^{3}$ School of Public Health, The University of Sydney, Sydney, New South Wales, Australia ${ }^{4}$ Cardiopulmonary Research, Mayo Clinic Scottsdale, Scottsdale, Arizona, USA ${ }^{5}$ Mayo Clinic Hospital Jacksonville, Jacksonville, Florida, USA

Correspondence to

Troy J Cross;

troy.cross@sydney.edu.au

\section{ABSTRACT}

There are growing concerns that the COVID-19 pandemic has facilitated a sedentary shift in our physical activity habits. A reduction in physical activity during the pandemic may be secondary to restrictive policies implemented at the government-level, typically those policies which limit interpersonal contact; for example, physical/social distancing. It is without question that social distancing is a necessary measure to mitigate community transmission of the novel virus; however, these policies often limit the public's opportunities to engage in physical activity. The strictest enforcement of social distancing occurs during an authority-mandated 'lockdown' (also known as a 'shelter-in-place' or 'stay-at-home' order). This Viewpoint focuses on the current evidence demonstrating that physical activity declines during a COVID-19 'lockdown'. We highlight the point that most of the available evidence stems from investigations using non-validated, selfreported measures of physical activity and discuss the caveats therewith. This Viewpoint explores whether current evidence reflects an 'actual' or rather a 'perceived' reduction in physical activity, and raises the question of whether this distinction matters in the end.

The coronavirus (COVID-19) outbreak was officially declared a pandemic on 11 March 2020 by the WHO. During the early phase of the COVID-19 pandemic, no effective pharmaceutical therapies existed to prevent and/ or contain the spread of the virus. Instead, authorities and governments around the world introduced several non-pharmaceutical interventions aimed at minimising the community spread of COVID-19. These interventions included directives for personal hygiene and respiratory etiquette, and public health measures to minimise interpersonal contact, such as social/physical distancing. ${ }^{1-3}$

There are concerns that social distancing has encouraged a shift towards a more physically inactive lifestyle. ${ }^{4} 5$ Indeed, many countries and local authorities have implemented social distancing via restrictions on domestic and international travel, closure of gymnasiums, sporting and recreational facilities, and/or through suspension of

\section{Key messages}

What is already known

- There are concerns that the COVID-19 pandemic has adversely impacted on the physical activity habits of the general population, particularly during authoritymandated 'lockdowns'.

- The majority of studies have quantified changes in physical activity during 'lockdowns' using nonvalidated, self-reported questionnaires.

- Prompting for self-reported physical activity from a time before a 'lockdown' likely confounds the evidence with recall bias.

\section{What are the new findings}

- This Viewpoint challenges the interpretation of published evidence, insofar as these findings may reflect a 'perceived' rather than 'actual' decline in physical activity during a COVID-19 'lockdown'.

team sports and activities that would otherwise require close interpersonal contact. Moreover, the workplace environment has changed dramatically since the beginning of the pandemic with large sections of the active workforce now 'working from home'. Under more extreme circumstances, government authorities have enforced stricter policies to limit interpersonal contact (curfews, ringfencing, etc) in the form of 'shelter-in-place', 'stay-at-home' or 'lockdown' orders. It is clear from the above that social distancing and authority-mandated 'lockdowns' drastically reduce the opportunities available for the public to engage in physical activity. At the time of writing, the pandemic is still current and social distancing remains one of the most effective methods to limit transmission of the disease. Despite recent emergency rollout and distribution of coronavirus vaccines around the world, it is likely that social distancing will be part of our 'COVID-19 normal' existence for the foreseeable future. If, as argued above, social distancing and recurrent 'lockdowns' favour the development of a sedentary lifestyle, we may yet see a longer term public

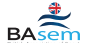


health issue that extends beyond the direct effects of the virus: a final wave of chronic lifestyle conditions may be waiting for us in the post-COVID world.

\section{CHANGES IN PHYSICAL ACTIVITY DURING A 'LOCKDOWN'}

There is no shortage of investigations examining the impact of COVID-19 'lockdowns' on physical activity. For example, a simple term search on PubMed using keywords of 'physical activity', 'COVID-19' and 'lockdown' returns 239 results since 2020 (search date: $5^{\text {th }}$ March 2021). Among these many results are preprints, original research, meta-analyses, systematic reviews and editorials. The consensus of this literature is that, indeed, physical activity declines during a COVID-19 'lockdown'. However, the notion that physical activity declines during a 'lockdown' presupposes that we know-with adequate confidence-the physical activity habits of the population before the 'lockdown' occurred.

Recently, Stockwell et at conducted a systemic review of all investigations that sought to determine changes in physical activity due to 'lockdowns' implemented throughout the COVID-19 pandemic. The overarching finding of this review was that physical activity declines and sedentary behaviour increases during a 'lockdown'-these observations are seemingly consistent across multiple populations (eg, children, adults, clinical patient groups) and countries. It is noteworthy that among the 66 studies included in their systematic review, only four investigations objectively measured physical activity using wearable devices before and during a 'lockdown' period (eg, accelerometers, pedometers, activity trackers, etc). The remaining studies assessed physical activity levels using self-reported questionnaires. That is, by asking participants to recall their physical activity habits during a current 'lockdown' and at a time before the 'lockdown' began. Importantly, however, of the studies using questionnaires to assess physical activity, the majority were non-validated $(n=39 / 59,66 \%)$. Given that it is exceedingly difficult to predict when a COVID-19 'lockdown' will occur, it is not surprising that most studies have relied on simple (although subjective) tools to assess physical activity in a retrospective, cross-sectional manner. It is however disconcerting that a large portion of the current evidence reporting a decline in physical activity during COVID-19 'lockdowns' stems from investigations using non-validated tools. This problem is further compounded by the larger issue of asking participants to recall events that occurred in their lives over relatively long windows of time.

Recall bias is the systematic error that occurs when participants do not accurately remember or omit details of past life events and experiences. As such, recall bias is a serious threat to the internal validity of any selfreported questionnaire-a threat which increases as the period of recall is extended further back into the past. It must be remembered that even standardised questionnaires typically ask participants to recall their physical activity over the 'previous 7 days' or during a 'typical week'-prompting for accurate information beyond this interval is tenuous. Further to this point, it is becoming clear that the on-going COVID-19 pandemic, and its many 'lockdowns', has adversely impacted on the mental health of the general public. ${ }^{7-9}$ It has been suggested that our ability to recall autobiographical events is influenced by our prevailing affective state (ie, mood-congruence recall bias). ${ }^{10}$ In this context, participants experiencing negative affect valence at the time of the survey may recall their physical activity habits prior to a COVID-19 'lockdown' with greater pessimism than those experiencing a more positive affective state.

\section{IS IT JUST A MATTER OF OPINION?}

The concerns outlined above raise the following questions: (i) What do bespoke (non-validated) surveys quantify if we are not certain of what they measure? (ii) How valid are standardised physical activity questionnaires when the period of recall is modified to refer to events occurring further in the past (ie, before a 'lockdown')? ${ }^{11}$ And finally: (iii) How might the mental health of a participant act to misconstrue or distort their recall of physical activity at any given point in time? At present, these questions do not have clear answers. Given that physical inactivity is already a pressing and serious concern for global health, ${ }^{512}$ it is important that we quantify the impact of the pandemic on physical activity habits as accurately as possible. Importantly, we do not suggest here that physical activity has not declined during the COVID-19 pandemic; rather, we argue that a conscious effort must be made by authors to acknowledge the specific limitations of their chosen self-reported measure of physical activity, such that we are appropriately cautioned to interpret the available evidence with consideration of the issues outlined above. Unless we can rule out the confounding effect of recall bias (and its moderators), we argue that the current literature may reflect a perceived rather than an actual change in physical activity during a COVID-19 'lockdown'. A participant's recollection of their own physical activity habits before a 'lockdown' may be less a matter of fact, and more a matter of opinion.

\section{DOES IT EVEN REALLY MATTER?}

It cannot be denied that, as Scientists, it behoves us to find empirical evidence to support our theses. Within the context of this Viewpoint, such evidence would be offered by more investigations reporting on objective measures of physical activity during a COVID-19 'lockdown'. Until this evidence is brought to light, we must reconcile ourselves with the fact that most of our knowledge on the topic is derived from subjective measures of physical activity during a 'lockdown'. But is that really a bad thing? If we reframe our thoughts, we see what is truly available to us: a large body of evidence showing that the general population perceives a decline in physical activity during a 'lockdown'. And perhaps it is this belief that physical activity has declined which determines 
health outcomes at present, and into the future. For example, a self-perceived reduction in physical activity during the COVID-19 pandemic is seemingly associated with poorer mental health outcomes, as evidenced by higher stress and anxiety. ${ }^{13}$ According to this perspective, new questions arise: Does a perceived reduction in physical activity during a 'lockdown' encourage future sedentary behaviour once the pandemic recedes? Do we observe better mental health outcomes during, and post-'lockdown' if we can alter such perceptions of decreased physical activity? Answering these questions will help us to determine whether the public's opinion of how the pandemic has affected our physical activity truly matters.

Twitter Troy J Cross @TroyJCross and Emmanuel Stamatakis @M_Stamatakis

Contributors All coauthors contributed to the conception, writing and editing of this work.

Funding The authors have not declared a specific grant for this research from any funding agency in the public, commercial or not-for-profit sectors.

Competing interests None declared.

Patient consent for publication Not required.

Provenance and peer review Not commissioned; externally peer reviewed.

Open access This is an open access article distributed in accordance with the Creative Commons Attribution Non Commercial (CC BY-NC 4.0) license, which permits others to distribute, remix, adapt, build upon this work non-commercially, and license their derivative works on different terms, provided the original work is properly cited, appropriate credit is given, any changes made indicated, and the use is non-commercial. See: http://creativecommons.org/licenses/by-nc/4.0/.

ORCID iD

Troy J Cross http://orcid.org/0000-0003-2902-7787

\section{REFERENCES}

1 Honein MA, Christie A, Rose DA, et al. Summary of guidance for public health strategies to address high levels of community transmission of SARS-CoV-2 and related deaths, December 2020. MMWR Morb Mortal Wkly Rep 2020;69:1860-7.

2 World Health Organiziation. Coronavirus disease (COVID-19) advice for the public. Available: https://www.who.int/emergencies/diseases/ novel-coronavirus-2019/advice-for-public

3 UK Government. Guidance: coronavirus (COVID-19): meeting with others safely (social distancing). Available: https://www.gov. uk/government/publications/coronavirus-covid-19-meeting-withothers-safely-social-distancing/coronavirus-covid-19-meeting-withothers-safely-social-distancing?priority-taxon=774cee22-d89644c1-a611-e3109cce8eae

4 Meyer SM, Landry MJ, Gustat J, et al. Physical distancing $\neq$ physical inactivity. Trans/ Behav Med 2021;11:941-4.

5 Hall G, Laddu DR, Phillips SA, et al. A tale of two pandemics: how will COVID-19 and global trends in physical inactivity and sedentary behavior affect one another? Prog Cardiovasc Dis 2021;64:108-10.

6 Stockwell S, Trott M, Tully M, et al. Changes in physical activity and sedentary behaviours from before to during the COVID-19 pandemic lockdown: a systematic review. BMJ Open Sport Exerc Med 2021;7:e000960.

7 Vindegaard N, Benros ME. COVID-19 pandemic and mental health consequences: systematic review of the current evidence. Brain Behav Immun 2020;89:531-42.

8 Pierce M, Hope H, Ford T, et al. Mental health before and during the COVID-19 pandemic: a longitudinal probability sample survey of the UK population. Lancet Psychiatry 2020;7:883-92.

9 Xiong J, Lipsitz O, Nasri F, et al. Impact of COVID-19 pandemic on mental health in the general population: a systematic review. J Affect Disord 2020;277:55-64.

10 Drace S. Evidence for the role of affect in mood congruent recall of autobiographic memories. Motiv Emot 2013;37:623-8.

11 Blair SN, Dowda M, Pate RR, et al. Reliability of long-term recall of participation in physical activity by middle-aged men and women. Am J Epidemiol 1991;133:266-75.

12 Kohl HW, Craig CL, Lambert EV, et al. The pandemic of physical inactivity: global action for public health. Lancet 2012;380:294-305.

13 Duncan GE, Avery AR, Seto E, et al. Perceived change in physical activity levels and mental health during COVID-19: findings among adult twin pairs. PLoS One 2020;15:e0237695. 